The authors wish to be identified to the reviewers.

2

\title{
Testosterone modulates status-specific patterns of cooperation in a
} 4 social network

6 Short title: Testosterone modulates cooperation

8 T. Brandt Ryder ${ }^{* \S}$, Roslyn Dakin ${ }^{\S}$, Ben J. Vernasco ${ }^{2}$, Brian S. Evans ${ }^{1}$, Brent M. Horton ${ }^{3}$, and Ignacio T. Moore ${ }^{2}$

10

${ }^{1}$ Migratory Bird Center, Smithsonian Conservation Biology Institute, National Zoological Park,

12 Washington, DC 20013, USA

${ }^{2}$ Department of Biological Sciences, Virginia Tech, Blacksburg, Virginia 24061, USA

$14{ }^{3}$ Department of Biology, Millersville University, Millersville, Pennsylvania 17551, USA

$16 \S$ Authors contributed equally

18 *Correspondence: rydert@si.edu 


\section{Summary}

Stable cooperation requires plasticity whereby individuals are able to express competitive or

22 cooperative behaviors depending on social context. To date, however, the physiological mechanisms that underlie behavioral variation in cooperative systems are poorly understood. We

24 studied hormone-mediated behavior in the wire-tailed manakin (Pipra filicauda), a gregarious songbird whose cooperative partnerships are crucial for fitness. We used automated telemetry to

26 monitor $>36,000$ cooperative interactions among male manakins over three field seasons, and we examined how circulating testosterone affects cooperation using $>500$ hormone samples.

28 Observational data show that in non-territorial floater males, high testosterone is associated with increased cooperative behaviors and subsequent ascension to territorial status. In territory-

30 holding males, however, both observational and experimental evidence demonstrate that high testosterone antagonizes cooperation. Moreover, circulating testosterone explains significant

32 variation (2-8\%) in social behavior within each status class. Collectively, our findings show that the hormonal control of cooperation depends on a male's social status. We propose that the

34 status-dependent reorganization of hormone-regulatory pathways can facilitate stable cooperative partnerships, and thus provide direct fitness benefits for males.

Keywords: cooperation, social networks, behavioral endocrinology, androgens, phenotypic 


\section{Introduction}

40 Behavioral plasticity is hypothesized to play a central role in the evolution of stable cooperation (Bergmuller et al. 2010; Díaz-Muñoz et al. 2014). For example, individuals must balance

42 competition for mating opportunities with the benefits of engaging in cooperation (CluttonBrock 2009; Cockburn 1998). These negotiations within cooperative social systems are mediated

44 by physiological mechanisms that govern behavioral plasticity (Soares et al. 2010) over the course of different temporal scales. Over the long-term, the plasticity that drives among-

46 individual differences in behavior and social status can facilitate stable cooperative partnerships (Bergmuller et al. 2010) as well as the competitive behaviors essential for establishing

48 dominance and acquiring status within social groups (Ryder et al. 2008, 2009). Over shorter timeframes, within-individual plasticity is thought to facilitate the expression of cooperative or competitive behaviors that are appropriate for the current social context (Taborsky and Oliveira 2012). To date, however, the proximate underpinnings of the behaviors that promote stable

52 cooperation are poorly understood (Soares et al. 2010). Thus, a key question is how physiological mechanisms modulate behavior in social systems where fitness requires a balance

54 of cooperative and competitive behaviors.

Steroid hormones are well known for their pleiotropic and often antagonistic effects on

56 numerous aspects of physiology and behavior (Ketterson et al. 1996; Wingfield et al. 2001; Adkins-Regan 2005; Hau 2007). Androgens, in particular, have been well studied in male

58 vertebrates, where they can either facilitate or constrain behavioral plasticity in reproductive and social contexts (Adkins-Regan 2005; Hau 2007). Yet, the vast majority of work on androgenmediated traits has focused on archetypal behaviors (e.g., aggression), with very few studies attempting to link hormones to individual variation in cooperation (Khan et al. 2001; Soares et 
62 al. 2010; Díaz-Muñoz et al. 2014). Although studies of cooperative social systems have documented status-specific differences in circulating testosterone levels (Wingfield et al. 1990;

64 Schoech et al. 1991; Peters et al. 2001; Ryder et al. 2011b), including the suppression of androgens in subordinates (Brouwer et al. 2009), no study has examined how androgens mediate the dynamics of cooperation and status within a social network.

Circulating hormones have long been recognized as a key mechanism that may underlie individual differences in behavior. Mounting evidence suggests that hormonal phenotypes (i.e., the expression of consistently low or high hormone levels) are often repeatable and may thus act as a source of heritable variation driving individual differences in behavior (Williams 2008; Mills et al. 2009; While et al. 2010; van Oers et al. 2011; Iserbyt et al. 2015). However,

72 empirical studies linking among-individual differences in circulating hormones to behavioral variation are challenging (Niemelä and Dingemanse 2018), in part, because hormone-mediated

74 behaviors are regulated by complex signaling pathways (Adkins-Regan 2005; Ball and Balthazart 2008; Rosvall et al. 2012; Fuxjager et al. 2018). Indeed, the complexity of steroid76 signaling pathways can produce nonlinear dose-dependent responses to circulating hormones, and these non-monotonic relationships may be key to understanding how hormones shape within 78 and among-individual differences in behavior (Lema 2014).

In addition to the above challenges, both steroid hormones and behavior are intrinsically 80 labile and responsive to the social environment. Steroids not only affect behavior, but they can also dynamically respond to it, and this social modulation of hormone-behavior relationships is 82 thought to optimize behaviors to the current social landscape (Wingfield et al. 1990; Goymann 2009; Oliveira 2009; Goymann et al. 2019). Engaging in social interactions can rapidly alter 84 circulating testosterone levels, androgen sensitivity in neural pathways, and the patterns of neural 
gene expression that influence motivation and shape status-specific behavioral phenotypes

86 (Burmeister et al. 2007; Maruska and Fernald 2010; Fuxjager et al. 2010). Transitions in social status can likewise alter circulating hormones and their impact on behavioral neural circuits

88 (Romeo et al. 2002; Maruska and Fernald 2010).

In an effort to expand our understanding of how hormones regulate social behavior, we

90 asked how circulating testosterone modulates cooperative behavior in a lek-breeding bird, the wire-tailed manakin (Pipra filicauda; Fig. 1A). Wire-tailed manakins have a social system with

92 two male status classes: territory-holders and floaters who do not yet hold a territory. Males from both classes form coalitions and perform a cooperative courtship display on territories at long-

94 established lek sites (Heindl 2002). These coalitions are important for reproductive success, as territorial males with more display partners sire more offspring, and floaters with more partners

96 have a higher probability of eventually inheriting a territory, which is a prerequisite for fitness (Ryder et al. 2008, 2009). Wire-tailed manakins do not exhibit overt aggression during display 2002; Ryder et al. 2008). As such, cooperative coalitions have clear fitness benefits, but they also

100 involve male-male competition and play a role in the ascension to territorial status. Many coalition partnerships are stable from year to year, but males also show substantial day-to-day

102 variation in their social interactions, creating a dynamic social network (Ryder et al. 2011a; Dakin and Ryder 2018). Previous work on this system has established that the status classes

104 differ in their circulating testosterone levels, with territory-holders having higher levels than floaters (Ryder et al. 2011b), and that cooperation is socially contagious (Dakin and Ryder 2018). 
Our goal in this study was to evaluate the role of a male's circulating testosterone in coalition partners, we utilized an autonomous behavior-logging system to monitor the social activity and interactions of 180 males (Ryder et al. 2012; Dakin and Ryder 2018). We focused on three behavioral phenotypes measured for each male on a daily basis (Fig. 1B): “effort”,

112 represents the duration of time a male spent attending the leks; "strength", his frequency of cooperative interactions; and "degree", his number of unique social partners. We consider effort

114 to be a measure of status seeking and status maintenance for floater and territorial males, respectively, whereas strength and degree are two measures of a male's cooperative tendencies.

116 In addition to repeated measures of behavior, we also collected repeated hormone samples from 210 individuals in our study population to characterize circulating testosterone levels over time;

118 each male was hormone sampled up to three times per field season (Fig. 1C). We used these hormone data to partition the standing variation in testosterone within and among-individuals, 120 while controlling for capture conditions (Williams 2008; Vernasco et al. 2019), and examined the relationships between testosterone and behavior.

Our analysis evaluates three predictions for the hypothesis that testosterone shapes male behavioral phenotype (Fig. 1D). First, given that testosterone plays a well-known role promoting 124 competitive ability and aggression in reproductive contexts (Goymann 2009), we consider that testosterone may be negatively related to cooperative behavior (i.e., "inhibition” in Fig. 1D). In

126 contrast, evidence in humans suggests that testosterone may promote reciprocity and fair bargaining (Eisenegger et al. 2010; Boksem et al. 2013). As such, our second prediction was that 128 testosterone may be positively related to cooperation (i.e., "facilitation" in Fig. 1D). Third, substantial evidence suggests that above some threshold, testosterone will no longer be 
correlated with behavior due to various mechanisms (Grunt and Young 1952; Kohn and Melnick 2002; Adkins-Regan 2005), or it may have detrimental effects on prosocial behaviors (e.g.,

132 parental care; Wingfield et al. 2001). As such, our third prediction was that testosterone may modulate cooperation in a nonlinear fashion (i.e., inverted U-function in Fig. 1D), whereby

134 individuals with intermediate circulating levels are the most cooperative. Given that wire-tailed manakins have two status classes, and that social status is known to modulate hormone-behavior relationships in other species (e.g., Maruska and Fernald 2010), our analysis also tested for status-dependence in the relationship between testosterone and behavior. We also examined the null hypothesis that social behavior may be decoupled from testosterone (i.e., "independence" in

Fig. 1D; Moore et al., 2004; Lynn et al., 2005; Hau, 2007). Finally, to establish a causal relationship between circulating testosterone and cooperation, we paired our observational analysis with a hormone manipulation experiment involving a subset of territorial males.

\section{Methods}

\section{Study System}

146 We studied a population of wire-tailed manakins (Pipra filicauda) that has been investigated and color-banded annually since 2002 at the Tiputini Biodiversity Station in Orellana Province,

148 Ecuador $\left(0^{\circ} 38^{\prime} \mathrm{S}, 76^{\circ} 08^{\prime} \mathrm{W}\right.$, approx. $200 \mathrm{~m}$ elevation). The breeding season lasts up to six months from November to April. Social status for each individual (i.e., territorial or floater) was

150 determined each year by direct observation (Ryder et al. 2008). Briefly, if an individual was seen displaying on a territory repeatedly and consistently over several days or weeks, he was assigned

152 as the territory owner. All blood samples and behavioral data were collected during peak 
breeding activity (December to March) over three field seasons (2015-16, 2016-17, and 2017-

154 18). Sample sizes were not predetermined, but rather we tried to capture and sample all males at the studied leks each field season.

156

\section{Testosterone Sampling and Assay}

158 Each male manakin was captured using mist-nets up to three times per field season. To maximize capture rates and minimize disturbance on the display territories (Vernasco et al. 2019), we

160 deployed up to 16 mist-nets simultaneously on a given lek. Nets were checked every 30 minutes.

A previous study demonstrated a subtle but significant effect net time (i.e., time from capture to

162 blood sampling) on circulating testosterone in manakins (Vernasco et al. 2019). Therefore, we used video monitoring of the mist nets to account for net time in our subsequent statistical

164 analyses (described below). The average span of time between repeated captures was 39 days (range $8-88$ days). Blood samples $(75-100 \mathrm{uL})$ were collected from the brachial vein and stored

166 on ice until return from the field when they were centrifuged for $5 \mathrm{~min}$ at 10,000 rpm. After centrifugation, plasma volume was measured to the nearest $0.25 \mathrm{ul}$ and stored in $0.75 \mathrm{ml}$ of $100 \%$

168 ethanol (Goymann et al. 2007); plasma volumes averaged 38.6uL (SD \pm 10.3uL). In the lab, testosterone was double extracted from plasma with dichloromethane (Ryder et al. 2011b). We used direct radioimmunoassay to determine the total plasma testosterone concentration $(\mathrm{ng} / \mathrm{mL})$, adjusted by extraction efficiency and by the plasma volume of the sample (Eikenaar et al. 2011).

172 Any sample that fell below the detection limit was assigned the assay-specific limit of detection as its testosterone concentration $(0.12,0.08$, and $0.09 \mathrm{ng} / \mathrm{mL}$ for $2015-16,2016-17$ and $2017-18$, 174 respectively). Extraction efficiency ranged from $62-73 \%$ and the intra-assay coefficients of 
variation were $6.6 \%, 11.6 \%$ and $9.2 \%$ for $2015-16,2016-17$ and $2017-18$, respectively. The inter-assay coefficient of variation was $19.5 \%$.

\section{Partitioning Variation in Testosterone}

A total of 524 testosterone samples were obtained from 210 individuals (1-8 samples per male;

Fig. 1C). To evaluate whether testosterone differed among individuals (Williams 2008; Hau and Goymann 2015), we fit a mixed-effects model of log-transformed circulating testosterone in the

182 lme4 package (Bates et al. 2018) in R (R Core Team 2018). The model included a bird's ID as a random effect and controlled for capture conditions that can influence hormone levels by using

184 fixed effects of field season (2015-16, 2016-17, or 2017-18), Julian date within the field season, time of day, and net time (i.e., see Vernasco et al., 2019). For 51 captures that were missing net

186 time data, we assumed the seasonal average net time (16-19 $\mathrm{min}$ ) to ensure that the missing values would not influence the analysis. We then computed the repeatability as the proportion of total variance in circulating testosterone explained by among-individual differences, after correcting for the fixed effects above. The $95 \%$ confidence intervals for repeatability were determined by parametric bootstrapping. To characterize the change in testosterone during social ascent, we added a fixed effect of four ascent categories to the same analysis (i.e., floaters who

192 did not subsequently ascend, floaters who subsequently acquired a territory, new territoryholders, and established territory-holders). We then used post-hoc Tukey tests and the

194 Benjamini-Hochberg procedure to compare status and ascent categories.

To examine hormone-behavior relationships, we used "corrected T", which we define as 196 the residuals from the linear model of log-transformed testosterone, after accounting for capture conditions (i.e., field season, Julian date, time of day, and net time). Next, we used within- 
subject centering to partition the variation in corrected $\mathrm{T}$ within- and among-individuals. The calculations followed the within-subject centering procedure in (van de Pol and Wright 2009); see Fig. A1 for a detailed illustration of this procedure. Briefly, we first computed each male's “mean T" as the average of his corrected T values. Next, we computed "relative T" by subtracting each male's mean $\mathrm{T}$ from his corrected $\mathrm{T}$ values. Hence, mean $\mathrm{T}$ is a variable that captures among-individual variation in testosterone (with one value of mean T per male). In

204 contrast, relative T represents a measure of a male's testosterone in a given blood sample, relative to his overall average (one value per blood sample).

\section{Social Behavior}

208 Social behaviors (Fig. 1B) were quantified on the display territories using an automated proximity data-logging system (described in Ryder et al. 2012; Dakin and Ryder 2018). Males

210 were outfitted with coded nano-tags (NTQB-2, Lotek Wireless; $0.35 \mathrm{~g}$ ), each of which transmitted a unique VHF signal ping once per 20 s (range: $19.5-20.5$ s). Approximately $10( \pm$ SD

212 7) days after capture, a proximity data-logger (SRX-DL800, Lotek Wireless) was placed within each territory at that lek to record social behaviors within a detection radius of approximately 30

214 m (i.e., the average diameter of a territory; Ryder et al. 2012; Dakin and Ryder 2018). The dataloggers recorded all tag pings emitted by males within each territory from 0600 to 1600 local

216 time for $\sim 6$ consecutive days ( $\mathrm{SD} \pm 1$ day). We attempted to recapture males at each lek for additional blood samples one to two more times per field season, after which social behaviors

218 were again recorded using the procedure described above. Thus, behaviors were monitored after hormone sampling, and hence we examine the relationship between testosterone levels and subsequent behavior. We recorded a total of 29,760 hours of social activity (2015-16: 49 
territories, mean $=16$ recording days per territory; 2016-17: 54 territories, mean $=21$ days per

222 territory; 2017-18: 48 territories, mean $=22$ days per territory, $\mathrm{SD}= \pm 4$ days in all field seasons). The number of males tagged for behavioral monitoring was $\mathrm{n}_{2015-16}=100, \mathrm{n}_{2016-17}=114$, and

$224 \mathrm{n}_{2017-18}=82$, for a total of $\mathrm{n}_{\text {tagged }}=180$ unique individuals. Note that the number tagged individuals is fewer than the number of blood-sampled males, because some males were captured 226 at unmonitored leks and not tagged. These additional individuals were included in the hormone repeatability and variance partitioning analysis to obtain more accurate estimates of each effect, 228 but they were not part of the behavioral study.

We defined three behavioral phenotypes. A male's effort, or his rate of lek attendance, 230 was quantified as his number of pings per day at monitored display territories. To define strength (frequency of cooperative interactions) and degree (number of unique social partners), the

232 proximity data were first filtered to identify times when two males co-occurred on a display territory as an indication of unique cooperative interactions (Ryder et al. 2008, 2012; Dakin and

234 Ryder 2018). A detailed description of the filtering algorithm is provided in (Dakin and Ryder 2018); a ground-truthing experiment in that study also confirmed that joint detections represent

236 individuals that were on average $<5 \mathrm{~m}$ apart. This spatial range corresponds to the visual and acoustic contact required for a typical display interaction (Heindl 2002). Extensive behavioral 238 observations of our study population have demonstrated that overt aggression (i.e., physical contact and chasing) is exceedingly rare on the display territories. As such, this automated 240 approach provides a reliable measure of cooperative interactions and increases both the quantity and quality of social network data relative to other methods (Ryder et al. 2012). An additional

242 validation study also confirmed that the social interactions defined by this automated system corresponded to cooperative display coalitions that were observed directly (Ryder et al. 2012). In 
244 total, we identified 36,885 unique social interactions over the present three-year study. Note that for floaters, our measures of effort, strength and degree included activity on any territory,

246 whereas for territory-holders, the same measures were limited to a male's activity on his own territory. Behavioral measures were transformed using $\log (\mathrm{x}+1)$ to meet the assumptions of

248 subsequent statistical analyses, and all measures were significantly repeatable (Dakin and Ryder 2018).

\section{Hormone Manipulation Experiment}

252 To test whether testosterone has direct effects on male behavior, we conducted a within-subjects hormone manipulation experiment on a subset of the territory-holding males. We chose the

254 territorial status class for this experiment because their high site fidelity makes them readily available for post-manipulation behavioral monitoring. Implant surgeries were performed on 14

256 randomly-selected territorial males during the peak of the breeding activity in two field seasons, 2016-17 and 2017-18. Subjects received a subcutaneous $7 \mathrm{~mm}$ length $\mathrm{x} 1.47 \mathrm{~mm}$ internal diameter

258 silastic implant sealed at both ends with $\sim 1 \mathrm{~mm}$ of silicone. The implants were either filled with crystalline testosterone ( $T$ treatment, $n=9$ ) or left empty (the control, $n=8$ ). Three of the males

260 who were manipulated in 2016-17 also received the opposite treatment in the subsequent field season $(n=17$ implants in total). Implant surgeries were scheduled between data-logger

262 recording sessions, such that each treatment male had a 6-day ( $\mathrm{SD} \pm 1$ day) pre-implant behavioral monitoring period within four weeks prior to implantation, and another 6-day (SD \pm 1

264 day) post-implant behavioral monitoring period within 10 days after implantation. We verified that the timing of pre- and post-implant behavioral monitoring did not differ between the two 
treatment groups (pre-monitoring, $\mathrm{p}=0.98$; post-monitoring, $\mathrm{p}=0.60$, Tukey's post-hoc tests on linear mixed-effects models).

15 days) for 17 implant surgeries to verify that the $T$ treatment significantly increased circulating

270 testosterone relative to pre-implant levels $(\mathrm{p}=0.04$; pre-implant least-squares mean from a mixed-effects model $=1.67 \mathrm{ng} / \mathrm{mL}[95 \% \mathrm{CI}=0.92,3.02]$; post-implant $=2.93 \mathrm{ng} / \mathrm{mL}[1.67$,

$2725.15])$. In contrast, the control implant did not significantly alter circulating testosterone $(\mathrm{p}=$

0.15 ; pre-implant $=1.21 \mathrm{ng} / \mathrm{mL}[95 \% \mathrm{CI}=0.55,2.66]$; post-implant $=0.57 \mathrm{ng} / \mathrm{mL}[0.26,1.25])$.

274 Note that although the least-squares mean was lower in the post-implant control group, it was still within the $95 \%$ CI of the pre-implant control group, indicating no significant change. We

276 also verified that all post-manipulation measures were within the natural (i.e., biologically relevant) range (see Fig. 1C and (Ryder et al. 2011b)). The average post-implant testosterone

278 level in the T treatment group corresponded to the $78^{\text {th }}$ percentile among unmanipulated territory-holding males.

\section{Statistical Analyses}

282 Prior to analyzing hormone-behavior relationships in the observational data, we removed data from individuals with a single blood sample, because quantifying relative T requires repeated

284 sampling (Appendix Fig. A1). The remaining measures of daily behavior were each assigned a relative T value using the bird's most recent prior hormone sample within four weeks (median 12 days, IQR 7-18 days). This step filtered the data to 2,363 daily recordings of 125 unmanipulated males. Next, we averaged the daily behavioral measures assigned to each blood sample (i.e., one row per unique measure of relative T). The final sample size was 346 hormone-behavior 
measures from 125 individuals, including 57 floaters and 76 territory-holders. Note that $56+$

$29076>125$, because some males were studied at both the floater and territory-holder stage.

To evaluate the hormone-behavior relationships in these data, we used a model selection

292 procedure in the MuMIn package (Bartoń 2018) in R (R Core Team 2018). Specifically, we evaluated support for competing models representing the hypotheses shown in Fig. 1D and

294 defined in Table A4. All candidate mixed-effects models included a male's identity as a random effect, as well as fixed effects of field season, Julian date within the field season, temperature

296 (daily averages acquired at the Tiputini Biodiversity Station), the number of days since capture, social status, and its interaction with testosterone (except in the null model, which did not

298 include testosterone). After the model selection procedure, statistical significance was determined using the lmerTest package (Kuznetsova et al. 2018) in R. In each case, we also tested for status-dependence of the hormone-behavior relationships by determining whether the interaction between status and a given testosterone metric was statistically significant. This

302 interaction tested whether the slope of the relationship differed between floater and territorial males. Given that we found significant status-dependence for all three behaviors, we next 304 repeated the model selection procedure for each status class separately. We present p-values from the best-fit model for each status class in our main results (see Appendix for complete 306 details on both sets of analyses). To determine the percent of behavioral variance explained by the testosterone metrics within each status class, we obtained $\mathrm{R}^{2}$ estimates using the $\mathrm{r} 2 \mathrm{glmm}$ 308 package (Jaeger 2017) in R (R Core Team 2018).

To analyze the effect of the hormone manipulation experiment on behavior, we fit mixed310 effects models that accounted for a male's identity as a random effect as well as fixed effects of field season, temperature, and implant (either pre-implant, control, or T treatment; $\mathrm{n}=211$ daily 
measures of 14 implanted individuals). We used post-hoc Tukey's tests in the multcomp package (Hothorn et al. 2017) in R (R Core Team 2018) to compare the treatments, and corrected p-

314 values using the step-up false discovery rate procedure in the multtest package (Pollard et al. 2018). We conducted a further analysis to determine if the effect of the T implant depended on a

316 bird's pre-implant hormone level. To do this, we compared the fit of models with, and without, the statistical interaction between a male's corrected $\mathrm{T}$ and the effect of the implant. We used

318 corrected $\mathrm{T}$ for this interaction because the small sample size in the experiment was not sufficient for variance-partitioning. The corrected $\mathrm{T}$ value came from the blood sample taken at 320 the time of surgery.

\section{Results}

Both the floaters and territory-holders expressed a broad range of testosterone levels (Fig. 2A,

324 Table 1). The floaters who successfully acquired a territory had significantly higher levels of circulating testosterone, as compared to other floaters who did not ascend ( $p=0.04$, Fig. 2B).

326 Importantly, testosterone levels were significantly repeatable in the population, with 19\% of the standing variation in hormone levels attributed to differences among-individuals. Thus,

328 repeatable differences in hormone levels have the potential to explain at least some of the among-individual differences in behavior.

Table 1. Descriptive statistics for metrics of circulating testosterone. Quartiles are provided

332 because the raw testosterone data have a log-normal distribution ${ }^{1}$. The sample size is $\mathrm{n}=524$ blood samples from 210 males (247 floater samples, and 277 territory-holder samples).

\begin{tabular}{|c|c|c|c|c|}
\hline Testosterone metric & Status class & $\begin{array}{l}25^{\text {th }} \\
\text { percentile }\end{array}$ & $\begin{array}{l}50^{\text {th }} \\
\text { percentile }\end{array}$ & $\begin{array}{l}75^{\text {th }} \\
\text { percentile }\end{array}$ \\
\hline
\end{tabular}




\begin{tabular}{lllll}
\hline Testosterone (ng/ml) & Floater & 0.17 & 0.37 & 1.21 \\
& Territory-holder & 0.63 & 1.62 & 2.56 \\
Corrected T (residual) & Floater & 0.23 & 0.48 & 1.61 \\
& Territory-holder & 0.73 & 2.01 & 3.47 \\
Mean T & Floater & -1.34 & -0.65 & 0.17 \\
\multirow{2}{*}{ Relative T } & Territory-holder & 0.03 & 0.37 & 0.89 \\
& Floater & -0.59 & -0.03 & 0.54 \\
& Territory-holder & -0.52 & 0.04 & 0.59 \\
\hline
\end{tabular}

We found that testosterone could explain significant variation in all three behaviors, but these hormone-behavior relationships were highly status-specific, as evidenced by the significant 38 slope differences between the two status classes (see Fig. 3). For floater males, the individuals with the highest levels of mean $\mathrm{T}$ attended the leks more often (effort) and engaged in cooperative interactions more frequently (strength; Fig. 3A-B). Floaters with higher mean T also tended to have more partners, although this relationship was not statistically significant (degree,

$342 \mathrm{p}=0.12$; Fig. $3 \mathrm{C}$ ). For territory-holding males, the males with higher mean $\mathrm{T}$ had significantly lower frequency of cooperative interactions (Fig. 3B), and tended to have fewer partners $(\mathrm{p}=$ 344 0.08; Fig. 3C). Lek attendance was highest in territory-holders with intermediate levels of mean T (Fig. 3A). In total, we found that the among-individual variation in testosterone (mean T)

346 could explain between $2-8 \%$ of the variation $\left(\mathrm{R}^{2}\right)$ in behavioral phenotypes within each status class (Fig. 3D). In contrast, the within-individual hormone variation (relative T) had a more

348 limited role, and was primarily related to one behavior, lek attendance (effort), but only in territory-holders (Fig. A2 in the Appendix). The highest level of lek attendance was observed

350 when a territory-holding male's circulating testosterone was closest to his average. Together with the result in Fig. 3A, this indicates that territory-holders reduce their lek attendance above or

352 below some threshold of mean $\mathrm{T}$ and relative $\mathrm{T}$. 
The territorial males with experimentally-elevated testosterone significantly decreased

354 their cooperative behavior, both in terms of the number of display partners (degree), and the frequency of cooperative interactions (strength) they received, although the manipulation did not

356 significantly affect their territory attendance (effort; Figure 4A-B). These experiment results are consistent with the correlative analysis (see Fig. 3), and confirm that testosterone levels above

358 some threshold have a direct negative impact on a territorial male's ability to maintain cooperative partnerships. Further analysis revealed that the effects of the T treatment also

360 depended on a male's pre-implant hormone level, as males with low testosterone prior to the implant lost more cooperative interactions and partners (strength and degree) than did males with

362 initially high pre-implant testosterone (Fig. 4C).

\section{Discussion}

The fitness of social animals is often contingent on the ability to express contextually relevant

366 behaviors in response to a dynamic social environment. In the social system of wire-tailed manakins, floater males must cooperate with territory-holders and compete with other floaters

368 for limited opportunities to acquire a territory (Ryder et al. 2008). However, once an individual ascends to territorial status, he must behaviorally shift his focus to attracting partners and

370 building stable coalition partnerships on his own territory, because females prefer more cooperative individuals (Ryder et al. 2009). As such, selection is expected to favor endocrine

372 mechanisms that promote behavioral plasticity to respond appropriately to conspecifics and ascension opportunities (Kelly and Vitousek 2017). Although we know that hormones can

374 coordinate both within- and among-individual plasticity in behavior and social competence (Dufty et al. 2002; Hau and Goymann 2015), the vast majority work on androgens has focused 
on how they facilitate courtship, aggression, competitive abilities, and dominance (Ketterson et al. 1996; Wingfield et al. 2001; Fusani 2008).

Here, we show that testosterone, an androgen long-known for its effect on aggression, can also influence social behavior in a cooperative system. Higher average testosterone (mean T) was associated with an increased frequency of cooperation in floaters (Fig. 3). Therefore, it does not appear to antagonize cooperation, but instead may facilitate the social behaviors necessary

382 for territory acquisition (Fig. 1D). Indeed, circulating testosterone was also predictive of which floaters ascended to territorial status, as the floaters that gained territories had higher testosterone 384 than those who did not obtain territories, both pre- and post-ascension (Fig. 2). Given that territory ownership and coalition partnerships are both necessary for a male to sire offspring in 386 this species (Ryder et al. 2008), these results suggest that high testosterone during the floater stage can yield later fitness benefits. Additional experiments are required to decouple the effects 388 of age and testosterone on these correlative results, because the floaters that successfully ascend the age-graded status queue also tend to be older. Experiments that manipulate hormones or 390 present social stimuli are also needed to determine whether higher testosterone causes statusseeking and cooperation in floaters, and/or whether testosterone rises in response to these 392 behaviors.

Once a male acquires a territory, his fitness depends on both the number of cooperative 394 partners and the stability of those partnerships (Ryder et al. 2009, 2011a). The results of our hormone manipulation experiment on territorial males confirmed that when testosterone in a territorial male is too high, it compromises his ability to attract and maintain stable display partnerships (Fig. 4). Hence, testosterone levels above some threshold at this stage could 398 decrease reproductive fitness. Our correlative data also support this hypothesis, as territorial 
males with lower mean $\mathrm{T}$ exhibited the optimal combination of social behaviors (i.e., high effort, strength, and degree in Fig. 3). Therefore, testosterone levels appear to be ultimately constrained in territory-holders despite their potential benefits at the floater stage. on a male's social status (Fig. 3), with all predictions in Fig. 1D represented, depending on the

404 behavior in question and status of the individuals. This finding suggests that a reorganization of the hormone-regulatory pathways involving testosterone may be required to optimize statusspecific social behaviors as males transition from floater to territorial. These changes may result from a number of physiological mechanisms that are not mutually exclusive. One possibility is that testosterone is only antagonistic to cooperative behavior above some concentration threshold that is not achieved by most floater males, and thus differential regulation of testosterone

410 secretion according to social status is essential. The threshold at which testosterone inhibits cooperation may also depend, in part, on an individual's hormonal phenotype or current

412 endocrine status. This possibility is supported by the fact that the negative effect of T-implants on cooperative behavior was greater for territory-holders with lower pre-implant testosterone

414 (Fig. 4C). Another possibility is that status-specific expression of steroidogenic enzymes and/or steroid receptors can cause among-individual and/or status-specific differences in the neural

416 sensitivity to testosterone (Romeo et al. 2002; Burmeister et al. 2007; Rosvall et al. 2012; Horton et al. 2014). Finally, testosterone can also have downstream effects on the release or actions of

418 other neurohormones known to influence social behavior, including steroid-sensitive neuropeptide systems (e.g., arginine vasotocin, and mesotocin; Kimura et al. 1999; De Vries and 420 Panzica 2006; Kabelik et al. 2010), and these pathways may vary between status classes. Each of these hypotheses represents a promising direction for identifying how variation in the hormone 
422 signaling pathways facilitates cooperation in the manakin lineage. Future work examining statusspecific neuroendocrine gene expression and its relationship with behavior in this species is a

424 pivotal next step.

Our repeated hormone sampling efforts also revealed that individual males differ

426 significantly in their circulating testosterone (corrected T; Fig. 1C). This finding of significant repeatability is consistent with growing evidence that hormonal phenotypes are often repeatable

428 and heritable in other systems (Williams 2008; Mills et al. 2009; While et al. 2010; van Oers et al. 2011; Iserbyt et al. 2015), yet robust estimates from wild populations, like those presented

430 here, are still exceedingly rare (Cox et al. 2016). Although the proportion of behavioral variation explained by among-individual variation in testosterone (mean T) was small at 2-8\% (Fig. 3D),

432 these $\mathrm{R}^{2}$ values are likely underestimates because of the measurement error associated with a limited number of repeat hormone samples (mean 2.5 blood samples per male, IQR 1-4; Fig.

434 1C). Moreover, a recent meta-analysis estimated that hormone levels generally account for only $2 \%$ of the variation in behavior, on average (Niemelä and Dingemanse 2018). Thus, the fact that 436 among-individual differences in circulating hormones can account for as much as $8 \%$ of the variation in the behavior of a free-living animal is an important result (Ball and Balthazart, 438 2008).

The social transmission of behaviors is hypothesized to play an important role in the 440 evolution of cooperation (Trivers 1971; Rankin and Taborsky 2009). Our previous work has established that manakin cooperative behaviors are indeed socially contagious, whereby the 442 cooperative behaviors of one individual stimulate subsequent cooperation in social partners (Dakin and Ryder 2018). Here, we provide evidence that testosterone can influence the 444 expression of socially-contagious behavior, in particular the frequency at which territory-holding 
males engage in cooperative interactions (Figs. 3-4). This raises the hypothesis for future study

446 that variation in testosterone levels may influence the collective structure and assortment of the social network. Because most coalition partnerships involve a territory-holder and a floater

448 (Ryder et al. 2011a), status-specific differences in how testosterone mediates behavior may promote a stable social network, by facilitating cooperation between males of different status 450 classes.

A recently proposed framework suggests that aggression and sociality are inextricably

452 linked and modulated by the same physiological and neural systems (Kelly and Vitousek 2017). In humans, the effects of testosterone depend on social status; testosterone can drive aggression

454 when individuals are socially challenged, but drive prosocial behaviors in the absence of those challenges (Boksem et al. 2013). Moreover in other vertebrate taxa, changes in social status may 456 often involve restructuring of the neural circuits and thus the way in which hormones mediate social behaviors (Romeo et al. 2002; Burmeister et al. 2007; Maruska and Fernald 2011;

458 Maruska 2015; Fernald 2015). In parallel with these discoveries, we show here that both the optimal level of testosterone and the way it modulates behavior may depend on a male's social 460 status. Overall, our findings support the hypothesis that status-dependent effects of androgens on social behavior may be a general feature of cooperative social systems where fitness depends on 462 behavioral plasticity. 


\section{References}

464 Adkins-Regan, E. 2005. Hormones and animal social behavior. Princeton University Press.

Ball, G. F., and J. Balthazart. 2008. Individual variation and the endocrine regulation of behaviour and physiology in birds: a cellular/molecular perspective. Philosophical Transactions of the Royal Society B: Biological Sciences 363:1699-1710.

468 Bartoń, K. 2018. MuMIn: multi-model inference.

470

472

474

476

478

480

482

484

486

488

490

492

494

496

498

500

502

504

Bates, D., M. Maechler, B. Bolker, S. Walker, R. H. B. Christensen, H. Singmann, B. Dai, et al. 2018. lme4: linear mixed-effects models using "Eigen" and S4.

Bergmuller, R., R. Schurch, and I. M. Hamilton. 2010. Evolutionary causes and consequences of consistent individual variation in cooperative behaviour. Philosophical Transactions of the Royal Society B 365:2751-2764.

Boksem, M. A. S., P. H. Mehta, B. Van den Bergh, V. van Son, S. T. Trautmann, K. Roelofs, A. Smidts, et al. 2013. Testosterone Inhibits trust but promotes reciprocity. Psychological Science 24:2306-2314.

Brouwer, L., T. G. G. Groothuis, O. Vedder, C. Eikenaar, D. S. Richardson, and J. Komdeur. 2009. Do primary males physiologically suppress subordinate males? An experiment in a cooperatively breeding passerine. Ethology 115:576-587.

Burmeister, S. S., V. Kailasanath, and R. D. Fernald. 2007. Social dominance regulates androgen and estrogen receptor gene expression. Hormones and Behavior 51:164-170.

Clutton-Brock, T. 2009. Cooperation between non-kin in animal societies. Nature 462:51-57.

Cockburn, A. 1998. Evolution of helping behavior in cooperatively breeding birds. Annual Review of Ecology and Systematics 29:141-177.

Cox, R. M., J. W. McGlothlin, and F. Bonier. 2016. Hormones as mediators of phenotypic and genetic integration: an evolutionary genetics approach. Integrative and Comparative Biology 56:126-137.

Dakin, R., and T. B. Ryder. 2018. Dynamic network partnerships and social contagion drive cooperation. Proceedings of the Royal Society B: Biological Sciences 285:20181973.

De Vries, G. J., and G. C. Panzica. 2006. Sexual differentiation of central vasopressin and vasotocin systems in vertebrates: different mechanisms, similar endpoints. Neuroscience, Neuroactive Steroids: Old Players in a New Game 138:947-955.

Díaz-Muñoz, S. L., E. H. DuVal, A. H. Krakauer, and E. A. Lacey. 2014. Cooperating to compete: altruism, sexual selection and causes of male reproductive cooperation. Animal Behaviour 88:67-78.

Dufty, A. M., J. Clobert, and A. P. Møller. 2002. Hormones, developmental plasticity and adaptation. Trends in Ecology \& Evolution 17:190-196.

Eikenaar, C., M. Whitham, J. Komdeur, M. van der Velde, and I. T. Moore. 2011. Endogenous testosterone is not associated with the trade-off between paternal and mating effort. Behavioral Ecology 22:601-608.

Eisenegger, C., M. Naef, R. Snozzi, M. Heinrichs, and E. Fehr. 2010. Prejudice and truth about the effect of testosterone on human bargaining behaviour. Nature 463:356-359.

Fernald, R. D. 2015. Social behaviour: Can it change the brain? Animal Behaviour 103:259-265.

Fusani, L. 2008. Testosterone control of male courtship in birds. Hormones and Behavior 54:227-233.

Fuxjager, M. J., R. M. Forbes-Lorman, D. J. Coss, C. J. Auger, A. P. Auger, and C. A. Marler. 2010. Winning territorial disputes selectively enhances androgen sensitivity in neural 
pathways related to motivation and social aggression. Proceedings of the National Academy of Sciences 107:12393-12398.

Fuxjager, M. J., M. C. Miles, and B. A. Schlinger. 2018. Evolution of the androgen-induced male phenotype. Journal of Comparative Physiology A 204:81-92.

Goymann, W. 2009. Social modulation of androgens in male birds. General and Comparative Endocrinology, 9th International Symposium on Avian Endocrinology 163:149-157.

Goymann, W., I. T. Moore, and R. F. Oliveira. 2019. Challenge hypothesis 2.0: a fresh look at an established idea. BioScience 69:432-442.

Goymann, W., I. Schwabl, M. Trappschuh, and M. Hau. 2007. Use of ethanol for preserving steroid and indoleamine hormones in bird plasma. General and Comparative Endocrinology 150:191-195.

Grunt, J. A., and W. C. Young. 1952. Differential reactivity of individuals and the response of the male guinea pig to testosterone propionate. Endocrinology 51:237-248.

Hau, M. 2007. Regulation of male traits by testosterone: implications for the evolution of vertebrate life histories. BioEssays 29:133-144.

Hau, M., and W. Goymann. 2015. Endocrine mechanisms, behavioral phenotypes and plasticity: known relationships and open questions. Frontiers in Zoology 12:S7.

Heindl, M. 2002. Social organization on leks of the wire-tailed manakin in southern venezuela. The Condor 104:772-779.

Horton, B. M., W. H. Hudson, E. A. Ortlund, S. Shirk, J. W. Thomas, E. R. Young, W. M. Zinzow-Kramer, et al. 2014. Estrogen receptor $\alpha$ polymorphism in a species with alternative behavioral phenotypes. Proceedings of the National Academy of Sciences 111:1443-1448.

Hothorn, T., F. Bretz, P. Westfall, R. M. Heiberger, A. Schuetzenmeister, and S. Scheibe. 2017. multcomp: simultaneous inference in general parametric models.

Iserbyt, A., M. Eens, and W. Müller. 2015. Sexually antagonistic selection during parental care is not generated by a testosterone-related intralocus sexual conflict-insights from full-sib comparisons. Scientific Reports 5:17715.

Jaeger, B. 2017. r2glmm: computes R squared for mixed (multilevel) models.

Kabelik, D., J. A. Morrison, and J. L. Goodson. 2010. Cryptic regulation of vasotocin neuronal activity but Not anatomy by sex steroids and social stimuli in opportunistic desert finches. Brain, Behavior and Evolution 75:71-84.

Kelly, A. M., and M. N. Vitousek. 2017. Dynamic modulation of sociality and aggression: an examination of plasticity within endocrine and neuroendocrine systems. Philosophical Transactions of the Royal Society B: Biological Sciences 372:20160243.

Ketterson, E. D., V. Nolan, M. J. Cawthorn, P. G. Parker, and C. Ziegenfus. 1996. Phenotypic engineering: using hormones to explore the mechanistic and functional bases of phenotypic variation in nature. Ibis 138:70-86.

Khan, M. Z., F. M. A. McNabb, J. R. Walters, and P. J. Sharp. 2001. Patterns of testosterone and prolactin concentrations and reproductive behavior of helpers and breeders in the cooperatively breeding red-cockaded woodpecker (Picoides borealis). Hormones and Behavior 40:1-13.

Kimura, T., K. Okanoya, and M. Wada. 1999. Effect of testosterone on the distribution of vasotocin immunoreactivity in the brain of the zebra finch, Taeniopygia guttata castanotis. Life Sciences 65:1663-1670. 
Kohn, M. C., and R. L. Melnick. 2002. Biochemical origins of the non-monotonic receptormediated dose-response. Journal of Molecular Endocrinology 29:113-123.

Kuznetsova, A., P. B. Brockhoff, and R. H. B. Christensen. 2018. lmerTest: tests in linear mixed effects models.

Lema, S. C. 2014. Hormones and phenotypic plasticity in an ecological context: linking

560

562

564

566

568

570

572

574

576

578

580

582

584

586

588

590

592

594

596 physiological mechanisms to evolutionary processes. Integrative and Comparative Biology 54:850-863.

Lynn, S. E., B. G. Walker, and J. C. Wingfield. 2005. A phylogenetically controlled test of hypotheses for behavioral insensitivity to testosterone in birds. Hormones and Behavior 47:170-177.

Maruska, K. P. 2015. Social transitions cause rapid behavioral and neuroendocrine changes. Integrative and Comparative Biology 55:294-306.

Maruska, K. P., and R. D. Fernald. 2010. Behavioral and physiological plasticity: rapid changes during social ascent in an African cichlid fish. Hormones and Behavior 58:230-240.

- 2011. Social regulation of gene expression in the hypothalamic-pituitary-gonadal axis. Physiology 26:412-423.

Mills, S. C., A. Grapputo, I. Jokinen, E. Koskela, T. Mappes, T. A. Oksanen, and T. Poikonen. 2009. Testosterone-mediated effects on fitness-related phenotypic traits and fitness. The American Naturalist 173:475-487.

Moore, I. T., H. Wada, N. Perfito, D. S. Busch, T. P. Hahn, and J. C. Wingfield. 2004. Territoriality and testosterone in an equatorial population of rufous-collared sparrows, Zonotrichia capensis. Animal Behaviour 67:411-420.

Niemelä, P. T., and N. J. Dingemanse. 2018. Meta-analysis reveals weak associations between intrinsic state and personality. Proceedings of the Royal Society B: Biological Sciences 285:20172823.

Oliveira, R. F. 2009. Social behavior in context: hormonal modulation of behavioral plasticity and social competence. Integrative and Comparative Biology 49:423-440.

Peters, A., L. B. Astheimer, and A. Cockburn. 2001. The annual testosterone profile in cooperatively breeding superb fairy-wrens, Malurus cyaneus, reflects their extreme infidelity. Behavioral Ecology and Sociobiology 50:519-527.

Pollard, K. S., H. N. Gilbert, Y. Ge, S. Taylor, and S. Dudoit. 2018. multtest: resampling-based multiple hypothesis testing.

R Core Team. 2018. R 3.5.1: A Language and Environment for Statistical Computing. R Foundation for Statistical Computing, Vienna, Austria.

Rankin, D. J., and M. Taborsky. 2009. Assortment and the evolution of generalized reciprocity. Evolution 63:1913-1922.

Romeo, R. D., H. N. Richardson, and C. L. Sisk. 2002. Puberty and the maturation of the male brain and sexual behavior: recasting a behavioral potential. Neuroscience \& Biobehavioral Reviews 26:381-391.

Rosvall, K. A., C. M. B. Burns, J. Barske, J. L. Goodson, B. A. Schlinger, D. R. Sengelaub, and E. D. Ketterson. 2012. Neural sensitivity to sex steroids predicts individual differences in aggression: implications for behavioural evolution. Proceedings of the Royal Society of London B: Biological Sciences rspb20120442.

Ryder, T. B., J. G. Blake, P. G. Parker, and B. A. Loiselle. 2011a. The composition, stability, and kinship of reproductive coalitions in a lekking bird. Behavioral Ecology 22:282-290. 
Ryder, T. B., B. M. Horton, and I. T. Moore. 2011b. Understanding testosterone variation in a tropical lek-breeding bird. Biology Letters 7:506-509.

Ryder, T. B., B. M. Horton, M. van den Tillaart, J. D. D. Morales, and I. T. Moore. 2012. Proximity data-loggers increase the quantity and quality of social network data. Biology Letters 8:917-920.

Ryder, T. B., D. B. McDonald, J. G. Blake, P. G. Parker, and B. A. Loiselle. 2008. Social networks in the lek-mating wire-tailed manakin (Pipra filicauda). Proceedings of the Royal Society B 275:1367-1374.

Ryder, T. B., P. G. Parker, J. G. Blake, and B. A. Loiselle. 2009. It takes two to tango: reproductive skew and social correlates of male mating success in a lek-breeding bird. Proceedings of the Royal Society B 276:2377-2384.

Schoech, S. J., R. L. Mumme, and M. C. Moore. 1991. Reproductive endocrinology and mechanisms of breeding inhibition in cooperatively breeding Florida scrub jays (Aphelocoma c. coerulescens). The Condor 93:354-364.

Soares, M. C., R. Bshary, L. Fusani, W. Goymann, M. Hau, K. Hirschenhauser, and R. F. Oliveira. 2010. Hormonal mechanisms of cooperative behaviour. Philosophical Transactions of the Royal Society B 365:2737-2750.

Taborsky, B., and R. F. Oliveira. 2012. Social competence: an evolutionary approach. Trends in Ecology \& Evolution 27:679-688.

Trivers, R. L. 1971. The evolution of reciprocal altruism. The Quarterly Review of Biology 46:35-57.

van de Pol, M., and J. Wright. 2009. A simple method for distinguishing within- versus betweensubject effects using mixed models. Animal Behaviour 77:753-758.

van Oers, K., K. L. Buchanan, T. E. Thomas, and P. J. Drent. 2011. Correlated response to selection of testosterone levels and immunocompetence in lines selected for avian personality. Animal Behaviour 81:1055-1061.

Vernasco, B. J., B. M. Horton, T. B. Ryder, and I. T. Moore. 2019. Sampling baseline androgens in free-living passerines: Methodological considerations and solutions. General and Comparative Endocrinology, Endocrinology of Neotropical Vertebrates 273:202-208.

While, G. M., C. Isaksson, J. McEvoy, D. L. Sinn, J. Komdeur, E. Wapstra, and T. G. G. Groothuis. 2010. Repeatable intra-individual variation in plasma testosterone concentration and its sex-specific link to aggression in a social lizard. Hormones and Behavior 58:208-213.

Williams, T. D. 2008. Individual variation in endocrine systems: moving beyond the 'tyranny of the Golden Mean.’ Philosophical Transactions of the Royal Society B: Biological Sciences 363:1687-1698.

Wingfield, J. C., R. E. Hegner, Dufty Alfred M., and G. F. Ball. 1990. The "challenge hypothesis": theoretical implications for patterns of testosterone secretion, mating systems, and breeding strategies. The American Naturalist 136:829-846.

Wingfield, J. C., S. E. Lynn, and K. K. Soma. 2001. Avoiding the 'costs' of testosterone: ecological bases of hormone-behavior interactions. Brain, Behavior and Evolution 57:239-251. 


\section{Acknowledgments}

We thank Camilo Alfonso, David and Consuelo Romo, Kelly Swing, Diego Mosquera, and

644 Gabriela Vinueza. This work was supported by the National Science Foundation (NSF) IOS 1353085, the Smithsonian Migratory Bird Center, the Global Change Center at Virginia Tech,

646 Millersville University, and the Tiputini Biodiversity Station of the Universidad San Francisco de Quito. Matt Fuxjager provided helpful feedback on an earlier version of this manuscript.

648

\section{Author contributions}

650 T.B.R., B.M.H., and I.T.M. designed the research

T.B.R., R.D., B.M.H., B.J.V., and I.T.M. performed the research

652 R.D., T.B.R., and B.S.E. analyzed the data

T.B.R. and R.D. wrote the manuscript

654 All authors edited the manuscript

656 Competing interests

The authors declare no competing interests.

658

\section{Additional information}

660 See the Appendix PDF for Figures A1-A2 and Tables A1-A9.

662 Data Availability

All materials necessary to reproduce the results including data and $\mathrm{R}$ code are available for

664 reviewers at the following link and will be made public upon acceptance:

https://figshare.com/s/d36c1a5c822e00abc581

666

\section{Ethics}

668 All methods were approved by the Smithsonian ACUC (protocols \#12-23, 14-25, and 17-11) and the Ecuadorean Ministry of the Environment (MAE-DNB-CM-2015-0008). 


\section{FIGURE LEGENDS}

672

Fig. 1. We studied how testosterone influences cooperative behavior in a social network. (A)

674 Wire-tailed manakins have a social system in which males of two status classes, territory-holders (black) and floaters (red), form cooperative display coalitions. Once a male attains territorial

676 status, he retains it for life. (B) We used an automated proximity system to quantify three social behaviors important for male fitness: effort is a measure of lek attendance; strength is the

678 frequency of cooperative interactions; and degree is the number of unique cooperative partners; each was measured on a daily basis. The violin plots show the distributions of individual means

$680(\mathrm{n}=180$ individuals $)$; the vertical lines are the medians. See Tables A1 and A2 in the Appendix for additional data. (C) We also collected repeated testosterone samples from 210 individuals

682 (inset; $\mathrm{n}=524$ blood samples). Analysis of these repeated measures revealed that circulating testosterone (T) varies both among- and within-individuals, allowing us to partition these two

684 sources of variation (Fig. A1 and Table A3 in the Appendix). The plot in (C) shows logtransformed testosterone for males with $>1$ hormone sample; each bar represents a single male, ordered along the x-axis by his average testosterone after correcting for capture conditions. Each individual's bar spans the $25^{\text {th }}$ and $75^{\text {th }}$ quantiles and is colored according to his status; the dark

688 line connects the medians. (D) We used these data to evaluates the predictions that testosterone may inhibit, facilitate, or have nonlinear (dose-dependent) effects on cooperation; the null hypothesis is that behavior is independent of circulating testosterone. We also considered that these hormone-behavior relationships may differ between the two status classes in (A). 


\section{Fig. 2. Elevated testosterone is associated with ascension to territorial status. (A)}

694 Histograms and probability density curves for log-transformed circulating testosterone in the two status classes ( $\mathrm{n}=524$ samples). (B) Change in corrected T during ascension to territorial status.

696 Floater males who acquired a territory the following field season had significantly higher testosterone than floaters who did not acquire a territory. The data points show partial residuals

698 from an analysis that controls for field season and capture conditions $(n=524$ measures from 210 individuals, including 15 who acquired territories during the study). Different letters indicate 700 statistically significant Tukey contrasts, after correcting for multiple comparisons.

Fig. 3. Social behavior is related to among-individual variation in circulating testosterone.

(A-C) Mean $\mathrm{T}$ is a measure of among-individual variation in circulating testosterone. Floaters

704 with the highest mean $\mathrm{T}$ had greater lek attendance (effort), cooperated with other males at higher frequencies (strength), and had more unique cooperative partners (degree), although the relationship with degree was not statistically significant $(n=57$ floaters). In contrast, territoryholding males with the highest mean T had lower lek attendance, a lower frequency of

708 cooperation, and fewer partners, although again the relationship with degree was not quite significant $(\mathrm{n}=76$ territory-holders). Each scatterplot shows the residuals (y-axis) from the best-

710 fit model after accounting for other predictors; note that all phenotypes were analyzed on a logscale. Red and grey asterisks indicate statistical significance within floaters and territory-holders,

712 respectively; the black asterisks compare hormone-behavior relationships between the two status classes (n.s. non-significant; ${ }^{*} \mathrm{p}<0.05 ; * * \mathrm{p}<0.01$ ). (D) The percent of behavioral variance

$714\left(\mathrm{R}^{2}\right)$ in each phenotype attributed to mean T. Further details are provided in Tables A4-A7 in the Appendix. 
Fig. 4. Experimental elevation of testosterone decreased cooperation in territory-holders.

718 Territorial males were given control $(n=8)$ or testosterone $(n=9)$ implants. (A) Average preand post-manipulation measures of lek attendance (effort), the frequency of cooperative

720 interactions (strength), and the number of partners (degree). Lines connect data from the same individual. The repeatability of each behavior, $\mathrm{R}$, is given above each panel. (B) Standardized

722 effect sizes from the statistical analysis (Control treatment, $\mathrm{C}$ post vs. pre; $\mathrm{T}$ treatment, $\mathrm{T}$ post vs. pre; and a comparison of the T treatment and the Control, T vs. C). Error bars show 95\%

724 confidence intervals. Experimentally-elevated testosterone decreased the frequency of cooperative interactions (strength) and the number of unique partners (degree), because T post 726 vs. pre and T vs. C were both statistically significant after correcting for multiple comparisons (n.s. non-significant; $* \mathrm{p}<0.05 ; * * \mathrm{p}<0.01 ; * * * \mathrm{p}<0.001$ ). The sample size for this analysis 728 was $n=211$ daily measures of 14 individuals. (C) Further analysis indicated that the effect of elevated testosterone on cooperative behavior (strength and degree) depended on the male's pre730 treatment corrected testosterone level. Specifically, stronger negative effects were observed for territory-holding males with lower pre-implant testosterone. See Tables A8-A9 in the Appendix

732 for additional data. Standardized effects sizes are shown to be comparable across the figure. 
bioRxiv preprint doi: https://doi.org/10.1101/453548; this version posted June 17, 2019. The copyright holder for this preprint (which was not certified by peer review) is the author/funder, who has granted bioRxiv a license to display the preprint in perpetuity. It is made available under aCC-BY-NC-ND 4.0 International license.

Fig. 1

A)

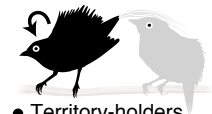

- Territory-holders

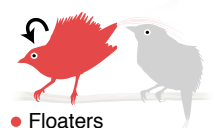

C)

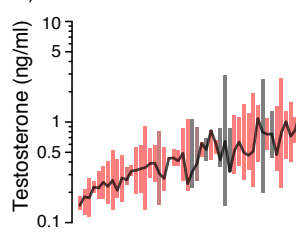

B)

Effort
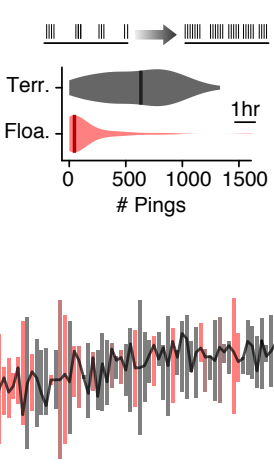
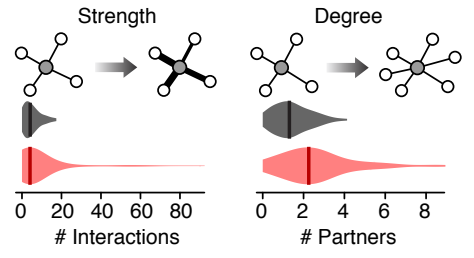

D) Inhibition Nonlinear

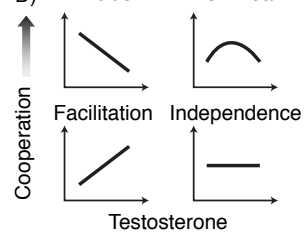


bioRxiv preprint doi: https://doi.org/10.1101/453548; this version posted June 17 2019. The copyright holder for this preprint (which was not certified by peer review) is the author/funder, who has granted bioRxiv a license to display the preprint in perpetuity. It is made available under aCC-BY-NC-ND 4.0 International license.

Fig. 2
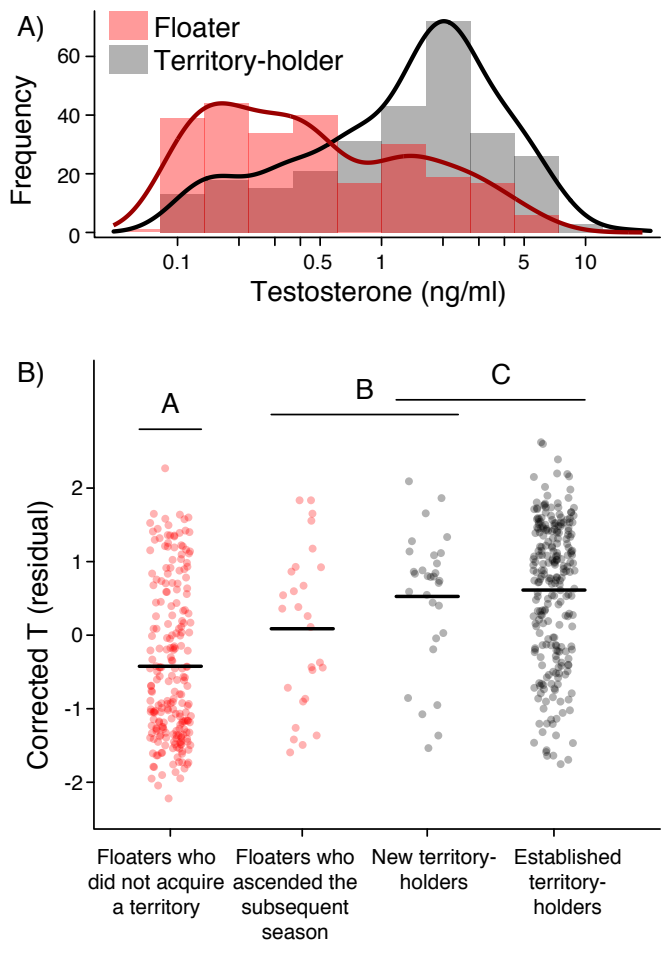
bioRxiv preprint doi: https://doi.org/10.1101/453548; this version posted June 17, 2019. The copyright holder for this preprint (which was not certified by peer review) is the author/funder, who has granted bioRxiv a license to display the preprint in perpetuity. It is made available under aCC-BY-NC-ND 4.0 International license.

Fig. 3
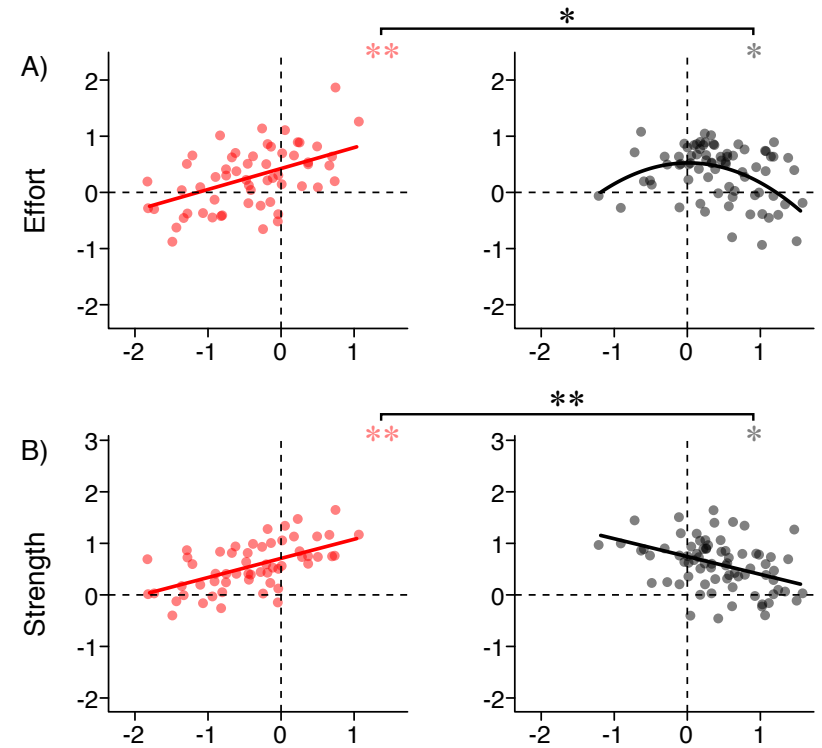

$* *$
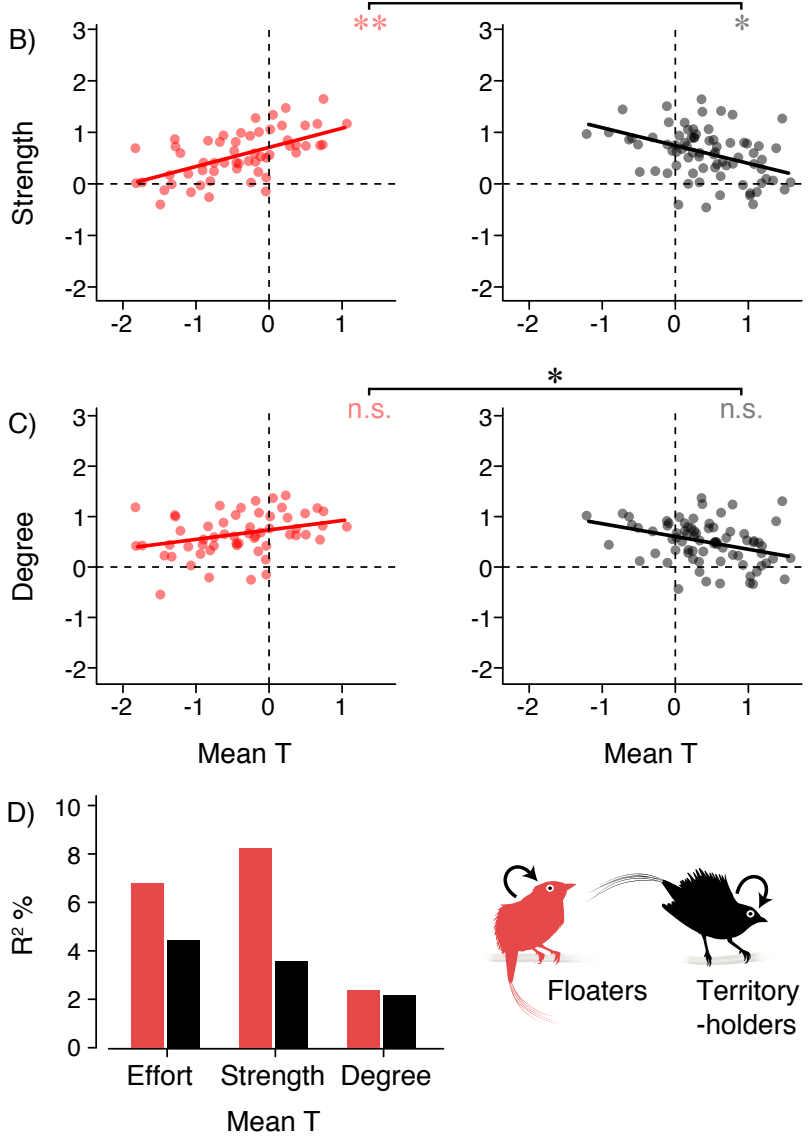
Fig. 4

A) Effort, $R=0.46$

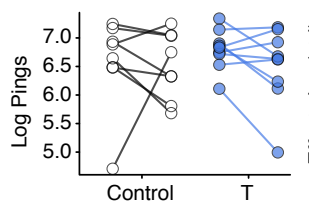

Strength, $R=0.54$

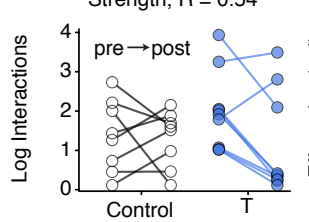

Degree, $R=0.47$

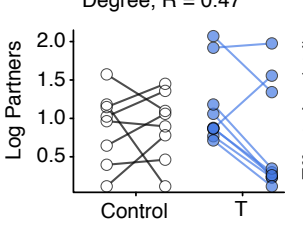

B)

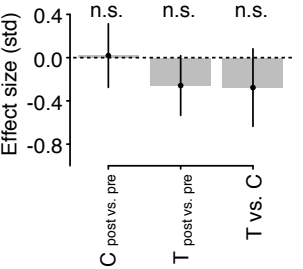

C)
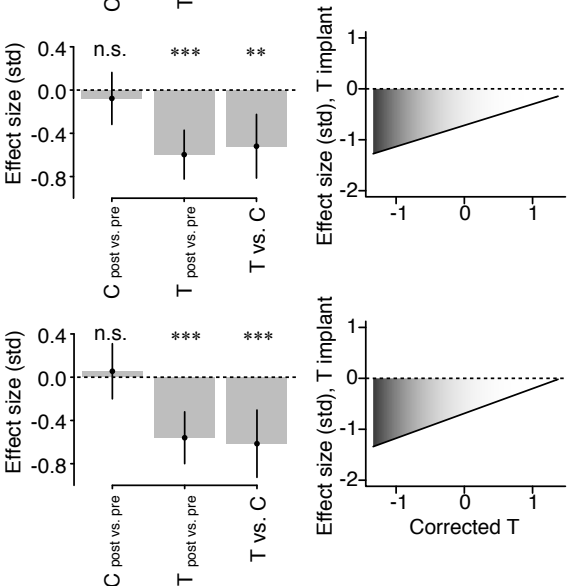